important that some form of evaluation should be incorporated into the programme, difficult though this may be.

"How can I tell if our efforts to introduce sterile procedures have had any effect on hospital infection rates?" asked a Danish nurse, speaking at a recent meeting on hospital twinning. "No reliable records were kept before we established our partnership with this hospital in Russia." It may be equally hard to measure the effect on morale of contact with peers in the West and access to modern managerial methods, technology, and publications.

Of course, it may be argued that many hospitals are more in need of heating and bedpans than lithotripters and computed tomography scanners, and clearly a balance has to be struck. What is exchanged needs to be relevant to the local needs, and links can be established at any level and thus reflect the concerns and priorities of all those providing care, from top management to ancillary workers.

Self sufficiency-not dependence-should be the aim. "Our need is not for your out of date drugs and equipment," said a Polish surgeon. "We want contact, and we can't afford to come to seminars in the West. We want interpretation as much as instruction. Teach us not only how to treat better but also how to treat more cost effectively. In exchange we can offer worthwhile collaboration, especially on research and development."

Britain has far fewer links in eastern and central Europe than most of its northern European neighbours, although arguably it has as much to offer-not least the experience of providing relatively low cost, effective, equitable, and humane medical care, and its strong teaching tradition. We need to come to terms with the fact that we are part of rather than adjacent to Europe and, irrespective of the recession, act accordingly.

Associate Editor, $B M \mathcal{F}$

TESSA RICHARDS

The editor of the newsletter of the Hospital Committee of the European Community may be contacted at Kapucijnenvoer 35, B-3000 Louvain, Belgium.

More information on twinning is available from Sir William Doughty, chairman of the North West Thames Regional Health Authority, who is head of the British delegation to the Hospital Committee of the European Community.

The Society for Cooperation and Development of Health Facilities and Structures (ACODESS) is at 7 rue du Fer à Moulin, 75005 Paris.

\title{
Can animals teach us medicine?
}

\section{Over the counter and into the forest}

The industrial world is increasingly realising that the tropics and their inhabitants still hold an irreplaceable, novel pharmacopoeia. As Fiona Godlee points out on p 1583 it awaits only better understanding for these compounds to be used in human medicine. ${ }^{12}$ Although a quarter of the drugs used in high tech medicine are derived from the natural world and our ancestors' experiments, blister packs and childproof caps disguise their origins. ${ }^{13+}$ Eight tenths of the world's population continues to rely on traditional medicines, but with the degradation of tropical forests and indigenous culture both the resources and the requisite knowledge face extinction.

Although analysis of the use of indigenous plants (ethnobotany) can point towards useful drugs, only $1 \%$ of the world's plants have been investigated.' Many drugs are now designed to act at specific biochemical sites rather than being found by screening or serendipity. ${ }^{5}$ Faced with the burden of human disease we should take advantage of alternative strategies. Modern drug design has to compete with millions of years of natural selection and coevolution. Returning to the field to watch animals and plants might provide useful insights.

The literature of tropical natural history is dotted with suggestions that vertebrates-especially monkeys, pigs, and elephants-use plants as medicines as well as food. ${ }^{6}$ The rainforest is a maze of toxins, foods, and medicines, requiring sophisticated use by animals and detailed study by zoologists. ${ }^{67}$ Animals' use of plant compounds, the study of sick wild animals, and examination of foraging patterns in health and disease may point the way to opening up animal medicine chests. ${ }^{7-9}$

Just like humans, animals seem to have discovered various routes of administration: they practise ingestion, buccal absorption, and topical application. Ethiopian baboons (Papio spp) at risk of schistosomiasis ingest Balanites fruits, which are rich in the potent antischistosome diosgenin, while those not exposed do not. ${ }^{10}$ Chimpanzees (Pan troglodytes) in
Tanzania may absorb potent chemicals from herbs, such as Aspilia, with a buccal feeding technique analogous to the human use of glyceryl trinitrate. They massage the leaf blade between their tongue and cheek before swallowing it whole. ${ }^{11-13}$ Aspilia, which is important in African traditional medicine, contains high concentrations of thiarubrine A, which has potent antifungal, antibacterial, and antinematode properties. The compound probably passes across the buccal mucosa but is destroyed by gastric acidity. ${ }^{12}$

Kodiak bears (Ursus arctos) apply Ligusticum porteri topically, and the Navajo Indians believe that their medical knowledge of Ligusticum came from the bears. Captive Kodiak bears chew, spit, and scratch the Ligusticum root into their fur; the root contains over 105 active compounds and may kill ectoparasites. ${ }^{14}$ A well studied but contentious case involves European starlings (Sturnus vulgaris), which combat parasitisation of their nests by incorporating specific "volatile" green vegetation close to incubating eggs. Whether fumigating the nest in this way improves the survival rate of hatchlings remains controversial. ${ }^{15}$

As well as using compounds prophylactically animals use drugs in active treatment. A lethargic, anorectic wild chimp with diarrhoea has been reported apparently treating itself with Vernonia shoot pith, which is used extensively in tropical Africa for gut disorders. ${ }^{16}$ Although most evidence points to antiparasitic functions, compounds used by animals may have other benefits. For example, howler monkeys (Alouatta) may use herbal medicines to control birth spacing and determine their offsprings' sex. ${ }^{1417}$ Koalas may consume Eucalyptus species differentially to aid thermoregulation; some species contain oils that raise, while others lower, body temperature. ${ }^{18}$

Some animals use herbal psychoactive compounds. Sloth bears (Melursus ursinus) and local people in the Central Indian Highlands become drunk through eating fermenting Madhuca flowers. ${ }^{19}$ Reindeer (Rangifer tarandus) become intoxicated through avidly consuming fly agaric mushrooms (Amanita muscaria). Lapps believe that, like the Navajo, they 
learnt of the plants' magical properties from their most familiar animal. ${ }^{20}$ Whether these mammals deliberately intoxicate themselves is unknown.

Harvesting sustainable plant products, including medicinal plants, can bring poor tropical countries higher economic returns than deforestation. ${ }^{21}$ Increasingly, people are realising that tropical forests are banks with potentially greater long term significance than their financial cousins. The number of possible therapeutic compounds is daunting, but animals may act as signposts, leading to important substances. Animals have practised medicine for millions of years: it would be tragic to lose their potentially lifesaving discoveries.

PAUL NEWTON

Senior house officer

Renal Unit,

Churchill Hospital,

Oxford OX3 7LJ

NATHAN WOLFE Research assistant

Palo Alto Center for Pulmonary Disease Prevention, 750 Welch Rd, Suite 200 .

Palo Alto, CA 94304-1509,

USA
1 Caldecott J. Medicine and the fate of tropical forests. BMF 1987;295:229-30

Reid WV Conserving life's diversity. Environmental Science \& Technology 1992:26:1090-5.

3 Farnsworth NR, Akerele O, Bingel AS, Soejarto DD, Guo Z. Medicinal plants in therapy. Bull World Health Organ 1985;63:965-81.

4 Hollman A. Plants in cardiology. London: BMJ, 1992

5 Freely J. New drugs. London: BMJ, 1991.

6 Janzen D. Complications in interpreting the chemical defenses of trees against tropical arboreal plant-eating vertebrates. In: Montgomery GG, ed. The ecology of arboreal folivores. Washington, DC: Smithsonian Institution, 1978:73-84.

Newton P. The use of medicinal plants by primates: a missing link? Trends in Ecology and Evolution 1991;6:297-9.

8 Sears C. The chimpanzee's medicine chest. New Scientist 1990 Aug 4:42-4

9 Carper ER, Dobson AP. What else can green monkeys tell us about AIDS? Trends in Ecology and Evolution 1987;2:374-6.

10 Phillips-Conroy JE. Baboons, diet and disease: food selection and schistosomiasis. In: Taub DM King FA, eds. Current perspective in primate social dynamics. New York: Van Nostrand Reinhol 1986:287-304.

11 Wrangham R, Nishida T. Aspilia spp leaves-a puzzle in the feeding behaviour of wild chimp: Primates 1983;24:276-82.

12 Newton P, Nishida T. Possible buccal administration of herbal drugs by wild chimpanzees ( $\mathrm{Pa}$ roglodytes) Animal Behaviour 1990;139:799-800.

13 Rodriguez E, Areguillin $M$, Nishida T, Uehara S, Wrangham R, Abramowski $Z$, et a Thiarubrine A, a bioactive constituent of Aspilia (Asteracea) consumed by wild chimpanzees. Experienta 1985;41:419-20.

14 Grisanzio JA. Fur-bearing pharmacists. Animals 1992;Sept/Oct: 26-30

5 Fauth PT. Ectoparasitism and the role of green nesting material in the European starling. Oecologia 1988;88:22-9.

16 Huffman $M$, Seifu $M$. Observations on the illness and consumption of a possible medicinal plan Vernonia amygdalina by a wild chimpanzee in Mount Mahale National Park. Primate 1989;30:51-63.

17 Lewin R. What monkeys chew to choose their children's sex. New Scientist 1992 Feb 22:15. 18 Eberhard IH. Ecology of the koala, Phascolarctos cinereus, in Australia. In: Montgomery GG, ed The ecology of arboreal herbivores. Washington, DC: Smithsonian Institution, 1978:315-27.

19 Brander AAD. Wild animals in central India. London: Edward Arnold, 1931

20 Phillips R. Mushrooms and other fungi of Great Britain and Europe. London: Pan, 1981

21 Prance G. Fruits of the rainforest. New Scientist 1990;125:42-5.

\section{Ghosts, visions, and voices}

\section{Sometimes simply perceptual mistakes}

\author{
The ghost sat down on the opposite side of the fireplace, as if he were \\ quite used to it. \\ "You don't believe in me," observed the Ghost. \\ "I don't," said Scrooge. \\ "What evidence would you have of my reality, beyond that of your \\ senses?" \\ "I don't know," said Scrooge. \\ "Why do you doubt your senses?" \\ "Because," said Scrooge, "a little thing affects them. A slight \\ disorder of the stomach makes them cheats. You may be an \\ undigested bit of beef, a blot of mustard, a crumb of cheese, a \\ fragment of an underdone potato. There's more of gravy than of grave \\ about you, whatever you are!"’
}

Hallucinations are not always pathological. Hearing one's name called aloud or hearing a person's voice but finding no one there is common. ${ }^{23} \mathrm{~A}$ few people describe hearing a comforting or advising voice at some time in their lives. ${ }^{2}$ Recently bereaved widows and widowers may "hear" or, more commonly, "see" their dead spouse. ${ }^{45}$ Up to one in six people in Britain and the United States have seen, heard, or otherwise experienced ghosts or spirits. ${ }^{6-8}$ Lights, visions, and voices may be seen or heard during profound religious or mystical experiences, especially conversion ${ }^{9}-$ the experiences of Joan of Arc and St Paul are familiar examples. ${ }^{10}$

Every culture has an explanation for voices and visions. For example, Islam understands all such experiences, including visions of saints and angels, clairvoyance, clairaudience, and out of body experiences, in terms of jinn (demons) deceiving humans. " Although psychiatrists would describe these experiences as hallucinations - that is, thoughts or images that the subject mistakenly attributes to an external reference - can they really be understood in terms of psychiatric syndromes?

As such experiences are transient and isolated, major psychiatric illness is unlikely. An overloaded digestive system - on which the unreformed Scrooge based his scepticism about the evidence of his senses and which the lone yachtsman
Slocombe blamed for the sight of an apparition after dining on plums and cheese ${ }^{12}-$ is perhaps insufficient cause for morE than a bad dream. But a transient psychosis induced by vitamin deficiency from fasting could cause brief hallucina tions. ${ }^{13}$ Religious and mystical visions could also arise from transient psychosis induced by pain or infection, perhap $\mathbb{Q}$ after self flagellation. ${ }^{13}$ Prolonged vigils may cause the illusions and hypnagogic hallucinations of profound sleep ${ }^{3}$ deprivation. ${ }^{14}$ Prolonged introspection may cause dissociative illusions and hallucinations. ${ }^{15}$ Cerebral dysfunction from any cause, including past head injury, ${ }^{16}$ may promote psychie experiences.

Ghosts that appear at night may be illusions-that is misinterpretations of real objects. Mistakes and misinterpre tations are easily made when there is little sensory stimula tion. "The yard was so dark that even Scrooge, who knew it\$ every stone, was fain to grope with his hands . . . Scrooge ${ }_{N}^{J}$ having his key in the door of the lock, saw in the knocker without its undergoing any intermediate process of change not a knocker, but Marley's face."

Ghosts may also arise from hypnagogic and hypnopompi\&్ hallucinations - that is, normal phenomena of "waking dreams," which occur during the period of clouded con sciousness that accompanies falling asleep and waking up. (A Scrooge woke his Ghost of Christmas Yet To Come "shrank, ? collapsed and dwindled down into a bedpost.") Seeing figureș or hearing music, chimes, or one's own name is a commorf experience. ${ }^{17}$

Hearing one's name called aloud may be a hypnagogic of hypnopompic hallucination or a misinterpretation of another sound. We have a low threshold for hearing our name an 8 therefore a strong tendency to misinterpret ambiguous souns as our name. But some people describe having this experience in clear consciousness, in silence. What may be happening here is that they mistakenly identify a very quiet sound, which would ordinarily be subliminal, for their name. 